\title{
Growing Bubbles Rising in Line
}

JOHN F. HARPER

john.harper@vuw.ac.nz

School of Mathematical and Computing Sciences Victoria University of Wellington, New Zealand

Over many years the author and others have given theories for bubbles rising in line in a liquid. Theory has usually suggested that the bubbles will tend towards a stable distance apart, but experiments have often showed them pairing off and sometimes coalescing. However, existing theory seems not to deal adequately with the case of bubbles growing as they rise, which they do if the liquid is boiling, or is a supersaturated solution of a gas, or simply because the pressure decreases with height. That omission is now addressed, for spherical bubbles rising at high Reynolds numbers. As the flow is then nearly irrotational, Lagrange's equations can be used with Rayleigh's dissipation function. The theory also works for bubbles shrinking as they rise because they dissolve.

\section{Introduction}

Consider bubbles rising in line in a liquid, with surface tension assumed large enough to keep them spherical, and surface activity small enough to ignore. If they remain the same constant size as they rise, their motion presents a problem simple enough to solve analytically to leading order, with computation needed only for such things as quadrature and solution of linear matrix equations, in two special cases: several bubbles in Stokes flow (Reynolds number vanishingly small) [6], and two bubbles at high Reynolds numbers [5], [7]. Computational fluid dynamics has also proved useful in this problem: Yuan \& Prosperetti [21] dealt with two spherical bubbles at various Reynolds numbers up to 200, and Zinchenko et al. [22] with two deformable bubbles or drops in Stokes flow. Experiments are mostly with lines of many bubbles, but Zinchenko et al. did find some with two, which confirmed their theory.

Bubbles that grow or shrink as they rise were studied in Stokes flow by Magnaudet \& Legendre [15] and in inviscid flow by Chincholle [4], though the methods needed are much older [1], [2], [3], [8]. C. A. Bjerknes wrote several papers on hydrodynamical action at a distance from 1868 onwards, which were summarised by Hicks [9] and published in book form by Bjerknes' son V. Bjerknes [3]. Herman [8] found the velocity potential and 
the kinetic energy for a pair of translating expanding spheres but did not evaluate the forces acting. Basset [1], [2] simplified Herman's method by expanding in series of spherical harmonics centred at the centres of the spheres instead of using exact closed-form image systems, but he did not consider simultaneous translation and expansion. Chincholle [4] did, but not to the order needed in the present work. Voinov \& Golovin [20] pioneered the use of Rayleigh's dissipation function with Lagrange's equations in irrotational bubble theory, and extended the proof to the case of bubbles of varying radius.

The present paper takes the analysis to higher order, unifying much previous work. The advantage of using Lagrange's equations is that the alternative, of assuming bubble velocities, finding the pressure by Bernoulli's theorem, and thence forces, requires more detail of flow in the wakes than is easy to find [16]. We deal first with the simplest non-trivial case: two bubbles many radii apart in a vertical line rising under gravity at high Reynolds number, for which irrotational flow is a good first approximation. The velocity of expansion (negative for a shrinking bubble) will be taken as given. In the real world, of course, it depends on whether a gas bubble is supersaturated or undersaturated, or whether vapour is evaporating or condensing, and on the diffusivities of both heat and gas in the liquid [13], [18], [19]. Some of the theory is then extended to more than two bubbles.

\section{Lagrangian Theory}

Voinov and Golovin [20] showed that if an infinite Newtonian viscous fluid contains $N$ spherical bubbles whose radii are $a_{j}, j=1, \ldots, N$ and whose centres are at positions determined by the $3 N$ coordinates $q_{i}, i=1, \ldots, 3 N$, and each bubble moves with Reynolds number $R_{j}$, and the flow is irrotational (a good approximation if every $R_{j} \gg 1$, and the total kinetic energy is $T\left(q_{i}, a_{j}, \dot{q}_{i}, \dot{a}_{j}\right)$, the total potential energy is $V\left(q_{i}, a_{j}\right)$, and the total rate of viscous dissipation is $D\left(q_{i}, a_{j}, \dot{q}_{i}, \dot{a}_{j}\right)$, then Lagrange's equations reduce to

$$
\frac{d}{d t}\left(\frac{\partial T}{\partial \dot{q}_{i}}\right)-\frac{\partial T}{\partial q_{i}}+\frac{\partial V}{\partial q_{i}}+\frac{1}{2} \frac{\partial D}{\partial \dot{q}_{i}}=0 .
$$

The errors are of order $R_{j}^{-1 / 2}$. Voinov and Golovin also gave the additional $N$ Lagrange equations involving variation of the bubble radii, and included the additional terms in them arising from the work that must be done on the fluid to expand a bubble, but we may safely ignore them because we take the velocity of expansion as given. 


\section{Irrotational Theory for Two Bubbles}

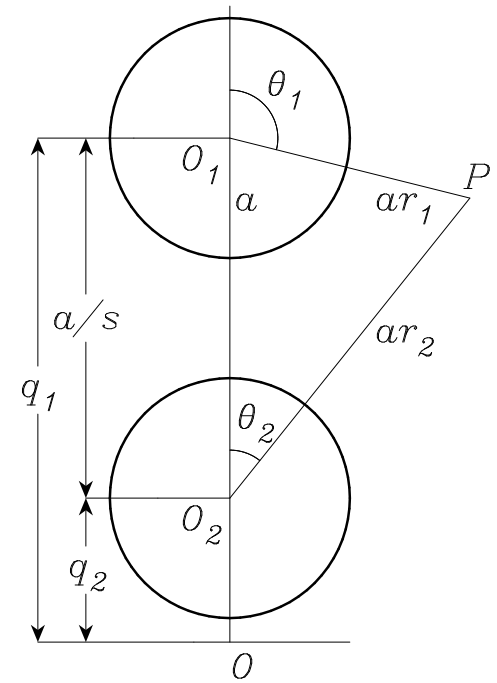

Figure 1. Two bubbles of the same radius $a$ rising in line, showing the dimensionless spherical coordinates $\left(r_{1}, \theta_{1}\right)$ and $\left(r_{2}, \theta_{2}\right)$ moving with each, the generalised coordinates $q_{1}, q_{2}$, and the definition of $s$. $O_{1}, O_{2}$ are the bubble centres, vertically above a fixed origin $O$.

Suppose that two gas bubbles are rising in the same vertical line with centres $O_{i}$ at heights $q_{i}$ above some fixed level, (see Figure 1), where $q_{1}>q_{2}$ and the liquid at a great distance is at rest. We may put $q_{3}=\cdots=q_{6}=0$ and use $i=j=1,2$. Let the distance $O_{1} O_{2}=q_{1}-q_{2}$ between the centres of the bubbles be $a / s$, where $s$ is dimensionless, and $s \rightarrow \frac{1}{2}$ for touching bubbles, $s \rightarrow 0$ for bubbles very far apart. Calculations will be presented only for $s \ll 1$, again for simplicity. Suppose also that the bubbles are in a liquid with dynamic viscosity $\eta=\rho \nu$, where $\rho$ is the density and $\nu$ the kinematic viscosity, with $\eta$ and $\rho$ much larger than in the gas in the bubbles, that surface activity is negligible, and the dimensionless number $M=g \eta^{4} / \rho \sigma^{3}$ is very small, where $g$ is the acceleration due to gravity and $\sigma$ is the surface tension, so that the Reynolds numbers $R_{i}=2 a \dot{q}_{i} / \nu$ can be (and are assumed to be) large while the bubbles remain close to a spherical shape (Moore [17]). In water, for example, $M$ is about $2.5 \times 10^{-11}$ and bubbles are nearly spherical if $R_{i}<400$. 
Let the bubbles have the same time-varying radius $a$, with surfaces $S_{i}$, let the expansion velocity be $\dot{a}=d a / d t$.

With the assumptions above, the flow is irrotational to leading order everywhere in the liquid [16], and in a frame of reference at rest in the liquid at a great distance it is generated by dipoles and sources at $O_{1}$ and $\mathrm{O}_{2}$, the images of each dipole and source in the other bubble, and so on (Herman [8], Basset [1], [2] chapter 11).

Let $P$ be any point in the fluid, let $P O_{i}=a r_{i}$, so that the $r_{i}$ are dimensionless, let $\theta_{i}$ be the angle between $P O_{i}$ and the upward vertical, and let $\cos \theta_{i}=\mu_{i}$. To order $s^{4}$, which is as far as one need go to find the leadingorder behaviour of the system, the velocity potential $\phi$ can be written as series $\phi_{1}\left(r_{1}, \theta_{1}\right)$ or $\phi_{2}\left(r_{2}, \theta_{2}\right)$ which converge near $S_{1}$ and $S_{2}$ respectively, and which are given by

$$
\begin{gathered}
\phi_{i}=-\frac{a}{2}\left[\frac{\dot{q}_{i} P_{1}}{r_{i}^{2}}+\dot{q}_{j}\left\{ \pm s^{2}-s^{3}\left(2 r_{i}+\frac{1}{r_{i}^{2}}\right) P_{1}+s^{4}\left(3 r_{i}^{2}+\frac{2}{r_{i}^{3}}\right) P_{2}\right\}\right. \\
+2 \dot{a}\left\{\frac{1}{r_{i}}+s \mp s^{2}\left(r_{i}+\frac{1}{2 r_{i}^{2}}\right) P_{1}+s^{3}\left(r_{i}^{2}+\frac{2}{3 r_{i}^{3}}\right) P_{2}\right. \\
\left.\left.+\frac{s^{4}}{2} \mp s^{4}\left(r_{i}^{3}+\frac{3}{4 r_{i}^{4}}\right) P_{3}\right\}\right]
\end{gathered}
$$

where $i=1$ or $2, j=3-i$, the upper ambiguous sign is for $i=1$, the lower one for $i=2, P_{n}$ denotes the Legendre polynomial $P_{n}\left(\mu_{i}\right)$, and the sign convention is that the velocity $\mathbf{v}=+\nabla \phi$.

Lamb [12], p. 46 and 580, gave the formulae

$$
\begin{aligned}
T & =-\frac{1}{2} \rho \iint_{S} \phi \frac{\partial \phi}{\partial n} d S, \\
D & =-\eta \iint_{S} \frac{\partial v^{2}}{\partial n} d S,
\end{aligned}
$$

where $v=|\mathbf{v}|$ is the speed of the fluid, occupying a region with boundary $S$ whose normal direction drawn into the fluid is $n$; equation (3) requires that $\phi \rightarrow 0$ at infinity, but the boundary $S$ need not be at rest. In our case $S$ consists of the surfaces of two bubbles in an unbounded fluid. Hence, to 
order $s^{4}$,

$$
\begin{aligned}
& T=\frac{\pi \rho a^{3}}{3}\left\{\dot{q}_{1}^{2}+\dot{q}_{2}^{2}+12(1+s) \dot{a}^{2}-\right. \\
& \left.\quad 6 s^{2} \dot{a}\left(\dot{q}_{1}-\dot{q}_{2}\right)-6 s^{3} \dot{q}_{1} \dot{q}_{2}+6 s^{4} \dot{a}^{2}\right\}, \\
& V=-\frac{4}{3} \pi \rho g a^{3}\left(q_{1}+q_{2}\right)+8 \pi \sigma a^{2}, \\
& D=12 \pi \eta a\left\{\dot{q}_{1}^{2}+\dot{q}_{2}^{2}+\frac{8}{3} \dot{a}^{2}-2 s^{2} \dot{a}\left(\dot{q}_{1}-\dot{q}_{2}\right)-4 s^{3} \dot{q}_{1} \dot{q}_{2}+2 s^{4} \dot{a}^{2}\right\},
\end{aligned}
$$

and Lagrange's equations for the $q_{i}(1)$ may be written in matrix form as

$$
\begin{gathered}
\left(\begin{array}{cc}
1 & -s^{3} \\
-s^{3} & 1
\end{array}\right)\left(\begin{array}{l}
\ddot{q}_{1} \\
\ddot{q}_{2}
\end{array}\right)+\frac{18 \nu}{a^{2}}\left(\begin{array}{cc}
1+\alpha / 6 & s^{3}(-2-\alpha) \\
s^{3}(-2-\alpha) & 1+\alpha / 6
\end{array}\right)\left(\begin{array}{l}
\dot{q}_{1} \\
\dot{q}_{2}
\end{array}\right) \\
+\frac{9}{a}\left(\begin{array}{cc}
0 & -s^{4} \\
+s^{4} & 0
\end{array}\right)\left(\begin{array}{l}
\dot{q}_{1}^{2} \\
\dot{q}_{2}^{2}
\end{array}\right)=\left(\begin{array}{l}
2 g+18 s^{2} \dot{a} \nu / a^{2}+9 s^{2} \dot{a}^{2} / a \\
2 g-18 s^{2} \dot{a} \nu / a^{2}-9 s^{2} \dot{a}^{2} / a
\end{array}\right),
\end{gathered}
$$

where $\alpha=\dot{a} a / \nu$ is a Reynolds number based on the expansion rate $\dot{a}$. We do not need the third Lagrange equation for the variable $a$ because we are assuming that $\dot{a}$ is given.

If there is just one bubble, and both gravity and viscosity are neglected, $T=\frac{1}{3} \pi \rho a^{3}\left(\dot{q}^{2}+12 \dot{a}^{2}\right), V=4 \pi \sigma a^{2}, D=0$, so that Lagrange's equations give $\partial T / \partial \dot{q}=M^{\prime} \dot{q}=$ constant, where $M^{\prime}=\frac{2}{3} \pi \rho a^{3}$, which is Chincholle's [4] result that the momentum associated with the virtual mass of the bubble is constant. A growing bubble would thus slow down, a shrinking one would speed up. That is what Chincholle's French text says (his equation 14 and Section 3), but his English abstract unfortunately says "An increase in volume accelerates it and a decrease in volume slows it down." before going on to describe the constancy of momentum.

If gravity and viscosity are not neglected, but the bubbles are of constant size and so far apart that their interactions can be neglected, $\dot{a}=0, s \rightarrow 0$, and Lagrange's equations reduce to

$$
\ddot{q}_{i}+\frac{18 \nu}{a^{2}} \dot{q}_{i}=2 g
$$

of which the general solution is

$$
\dot{q}_{i}=u+w_{i} \exp \left(-18 \nu t / a^{2}\right),
$$

where $u=a^{2} g / 9 \nu$ is the terminal velocity of an isolated spherical bubble at high Reynolds number $R=2 u a / \nu, w_{i}$ are arbitrary constant velocities, and $a^{2} / 18 \nu$ is the "relaxation time" required for the difference between the speed $\dot{q}_{i}$ and $u$ to decrease by a factor $e$. 
If we assume that the accelerations $\ddot{q}_{i}$ are negligible, and that $s^{4} \dot{q}_{i}^{2}$ may be replaced by $s^{4} u^{2}$ because $s \ll 1$ and $\dot{q}_{i}$ is not far from $u$, equation (8) reduces to

$$
\dot{q}_{i}=f(\alpha)\left\{u \pm s^{2} \dot{a}\left(1+\frac{1}{2} \alpha\right)+2 s^{3} u\left(1+\frac{1}{2} \alpha\right) f(\alpha) \pm \frac{1}{4} s^{4} u R\right\},
$$

where $f(\alpha)=1 /\left(1+\frac{1}{6} \alpha\right)$ and \pm is interpreted as before: + for $i=1$, - for $i=2$. The term $\pm s^{2} \dot{a}$ implies that in the absence of other effects each bubble would move away from the other as if in the velocity field of the source at the centre of that other, if $\alpha$ is so small that $1+\frac{1}{6} \alpha$ is close to 1 . The next term $\pm s^{2} \dot{a} \alpha / 2= \pm s^{2} \dot{a}^{2} a /(2 \nu)$ is the effect on the bubble velocities of the Bjerknes force, which in this problem is a repulsion between the bubbles even if both are growing: the attraction usually expected in that case is for bubbles whose radius oscillates sinusoidally. The next term in (9), involving $s^{3} u$, does for the dipole images what $\pm s^{2} \dot{a}$ did for the sources, with the intriguing differences that the bubbles are both speeded up by the same amount, and by twice the velocity that would have been expected if the effect was purely kinematic and $\alpha=0$, but by a factor $2\left(1+\frac{1}{2} \alpha\right) /\left(1+\frac{1}{6} \alpha\right)^{2}$ in general. The final term, $\pm \frac{1}{4} s^{4} u R$, is the effect on the velocities of the inverse fourth power repulsion found by Harper [5]. It was previously given, with the wrong sign, by Jeans [10], p. 360, as an example on Lagrangian mechanics where the formula for kinetic energy was given, but in a form which is valid only for two spheres with the velocity of each being positive if towards the other. Unfortunately one needs to take the same direction along the line as positive for all the bubbles.

The upper bubble always rises faster than than the lower one according to this theory, unless the bubbles are shrinking so fast that $|\dot{a}|>2 s u\left(1+\frac{1}{2} \alpha\right)$. The forces on the bubbles are not equal and opposite because d'Alembert's paradox does not apply to unsteady flow.

\section{Other Effects}

The foregoing theory gives the leading-order effect of each bubble on the other subject to the assumptions there stated. As bubbles are often observed coalescing, some other physical mechanism must be operating besides those already considered. Obvious candidates are distortion from spherical shape, effects due to more than two bubbles, surface activity, and the viscous wake of upper bubbles affecting the rise of bubbles beneath them.

When the Weber number $2 \rho u^{2} a / \sigma$ is small enough for bubbles to be still very nearly spherical, distortion cannot be important. 
If there are more than two bubbles, the image dipoles do not lead to equal upwards velocities as they do for two, because the intermediate ones have dipoles nearby both above and below them, but the top and bottom ones do not. The kinetic and potential energies and the dissipation function can all be calculated by the previous methods; if $\dot{a}=0$ the result is, to leading order,

$$
\begin{aligned}
& T=\frac{1}{3} \pi \rho a^{3} \sum_{i}\left(\dot{q}_{i}^{2}-6 \sum_{j>i} s_{i j}^{3} \dot{q}_{i} \dot{q}_{j}\right), \\
& V=-\frac{4}{3} \pi \rho g a^{3} \sum_{i} q_{i}+4 N \pi \sigma a^{2}, \\
& D=12 \pi \eta a \sum_{i}\left(\dot{q}_{i}^{2}-4 \sum_{j>i} s_{i j}^{3} \dot{q}_{i} \dot{q}_{j}\right) .
\end{aligned}
$$

where $s_{i j}=a /\left(q_{i}-q_{j}\right)$, and $N$ is the number of bubbles.

For three equally spaced bubbles, $s_{12}=s_{23}=s, s_{13}=\frac{1}{2} s$, the upward velocities (reading down the line) are

$$
\begin{aligned}
& \frac{9}{4} s^{3} u+\frac{17}{64} s^{4} R u, \\
& 4 s^{3} u, \\
& \frac{9}{4} s^{3} u-\frac{17}{64} s^{4} R u .
\end{aligned}
$$

The second bubble always rises faster than the third, and it will tend to catch up the first if $R s<112 / 17=6.588$, but that condition requires that at large Reynolds number $R$ the bubbles must be a very long way apart.

With a line of many bubbles, the general conclusion will still hold but the detailed numbers would of course change. The second bubble will still tend to catch up the first, and then they will rise faster than the third. If they do not coalesce, bubbles 3 and 4 will then behave like bubbles 1 and 2 , and then bubbles 5 and 6 will, and so on; such pairing off is indeed sometimes seen in lines of bubbles [11].

In liquids which are contaminated but are so nearly pure that the bubbles have only small stagnant caps, the second one rises slower than the first at a low Reynolds number [14], as it would at a high Reynolds number if the caps were small enough to have their own Reynolds numbers small. However in more polluted liquids the bubbles behave like rigid spheres, for which the drag and hence the effect of the wake are larger.

The most likely cause of bubble coalescence in pure liquids is then the effect of the viscous wake analysed by Katz and Meneveau [11] and Harper [7], in which a bubble rises faster if in the fluid rising in the viscous wake of 
a preceding one, especially in experiments using polluted liquids or more than two bubbles. However, the results of this paper cast doubt on the detailed mathematics of this part of [11] and [7], because even in irrotational flow the additional speed of rise of a bubble, due to the motion of the other bubbles, is not what one might expect.

\section{Conclusions}

Expansion of bubbles due to supersaturation or boiling does not of itself remove the discrepancy between experiment (in which bubbles in line often coalesce) and most theory (in which they seem not to if the Reynolds number is high). Shrinkage due to undersaturation or condensation can make bubbles move closer together, and in a polluted liquid the second bubble can climb up the wake of the first, in which the liquid is already rising, to achieve the same result. However the present work shows that this wake-climbing effect may not just be the mean across bubble $n$ of the wake speed behind bubble $n-1$ suggested by Katz and Meneveau [11]: that property is only approximately true for the source flow due to expanding bubbles, and it suggests about half the correct result for the image dipole contributions. The subject clearly needs more work.

\section{Acknowledgments}

I am grateful for financial support to the Marsden Fund administered by the Royal Society of New Zealand, and for hospitality while working on this subject to the Oxford Centre for Industrial and Applied Mathematics, St. Catherine's College, Oxford, the Department of Mathematics, Heriot-Watt University, Edinburgh, and the Department of Applied Mathematics and Theoretical Physics, Cambridge.

\section{References}

1. A. B. Basset. On the motion of two spheres in a liquid, and allied problems. Proc. Lond. Math. Soc., 18:369-377, 1887.

2. A. B. Basset. A treatise on hydrodynamics with numerous examples, volume 1. Deighton Bell, Cambridge, 1888. Reprinted 1961 by Dover, New York.

3. V. Bjerknes. Vorlesungen über hydrodynamische Fernkräfte nach C. A. Bjerknes' Theorie. J. A. Barth, Leipzig, 1900-1902. 2 vols.

4. L. Chincholle. Étude de l'écoulement d'une émulsion. Première partie. Étude théorique du mouvement d'une bulle. Effet fusée. Pébullon. La Houille Blanche, 22:515-529, 1967. 
5. J. F. Harper. On bubbles rising in line at large Reynolds numbers. J. Fluid Mech., 41:751-758, 1970.

6. J. F. Harper. Axisymmetric Stokes flow images in spherical free surfaces, with applications to rising bubbles. J. Austral. Math. Soc. Ser. B. Appl. Math., 25:217$231,1983$.

7. J. F. Harper. Bubbles rising in line: why is the first approximation so bad? J. Fluid Mech., 351:289-300, 1997.

8. R. A. Herman. On the motion of two spheres in fluid and allied problems. $Q$. $J l$ Pure Appl. Math., 22:204-262, 1887.

9. W. M. Hicks. Report on recent progress in hydrodynamics. Rep. Brit. Assn. Adv. Science, 52:39-70, 1882.

10. J. H. Jeans. An elementary treatise on theoretical mechanics. Ginn \& Company, Boston, 1907.

11. J. Katz and C. Meneveau. Wake-induced relative motion of bubbles rising in line. Int. J. Multiphase Flow, 22:239-258, 1996.

12. H. Lamb. Hydrodynamics. Cambridge University Press, London and New York, 6th edition, 1932.

13. D. Legendre, J. Borée, and J. Magnaudet. Thermal and dynamic evolution of a spherical bubble moving steadily in a superheated or subcooled liquid. Phys. Fluids, 10:1256-1272, 1998.

14. L. Lerner and J. F. Harper. Stokes flow past a pair of stagnant-cap bubbles. J. Fluid Mech., 232:167-190, 1991.

15. J. Magnaudet and D. Legendre. The viscous drag force on a spherical bubble with a time-dependent radius. Phys. Fluids, 10:550-554, 1998.

16. D. W. Moore. The boundary layer on a spherical gas bubble. J. Fluid Mech., 16:161-176, 1963.

17. D. W. Moore. The velocity of rise of distorted gas bubbles in a liquid of small viscosity. J. Fluid Mech., 23:749-766, 1965.

18. E. Ruckenstein. On heat transfer between bubbles in motion and the boiling liquid from which they are generated. Chem. Eng. Sci., 10:22-30, 1959.

19. L. E. Scriven. On the dynamics of phase growth. Chem. Eng. Sci., 10:1-13, 1959.

20. O. V. Voinov and A. M. Golovin. Lagrange equations for a system of bubbles of varying radii in a liquid of small viscosity. Fluid Dynamics, 5:458-464, 1970.

21. H. Yuan and A. Prosperetti. On the in-line motion of two spherical bubbles in a viscous fluid. J. Fluid Mech., 278:325-349, 1994.

22. A. Z. Zinchenko, M.A. Rother, and R. H. Davis. Cusping, capture and breakup of interacting drops by a curvatureless boundary-integral algorithm. J. Fluid Mech., 391:249-292, 1999. 


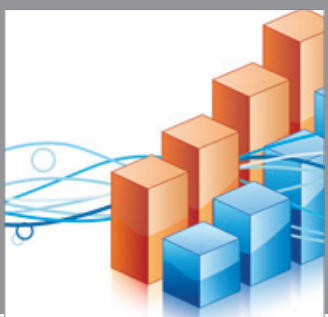

Advances in

Operations Research

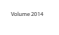

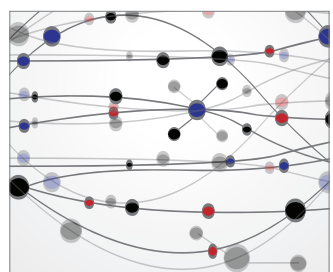

\section{The Scientific} World Journal
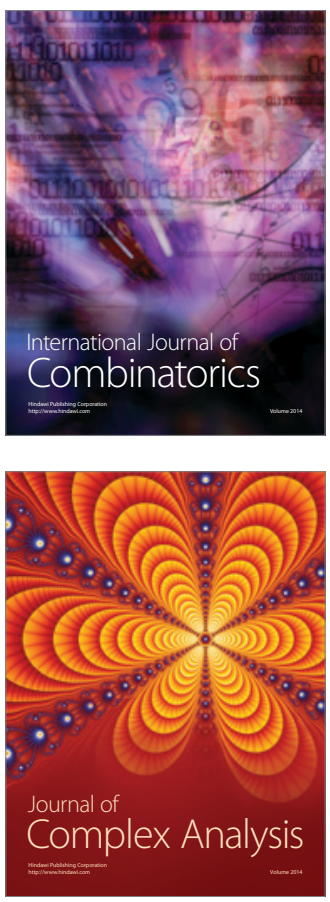

International Journal of

Mathematics and

Mathematical

Sciences
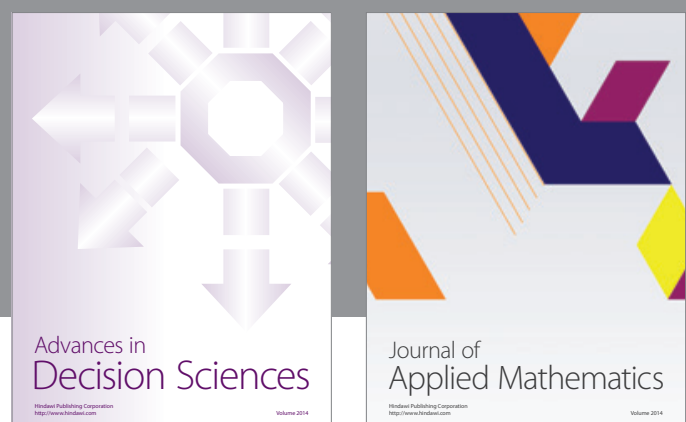

Journal of

Applied Mathematics
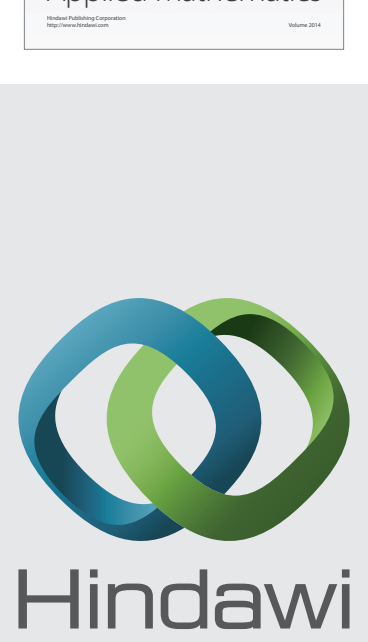

Submit your manuscripts at http://www.hindawi.com
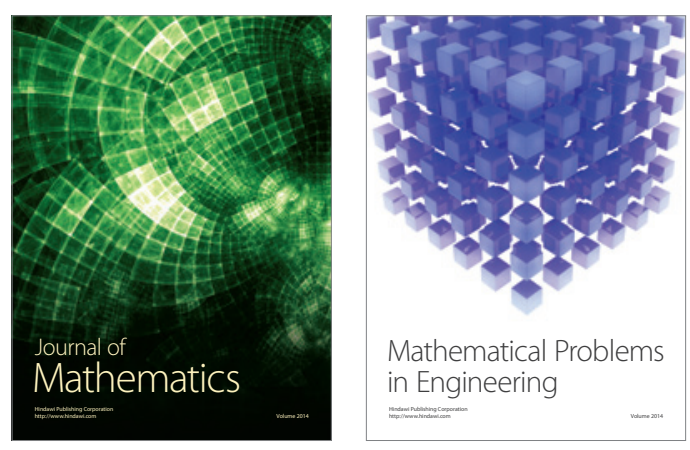

Mathematical Problems in Engineering
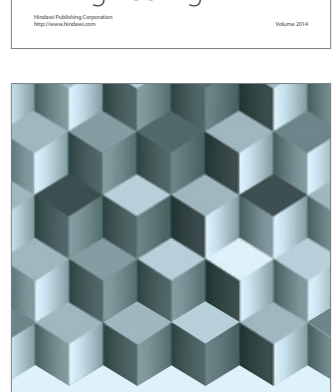

Journal of

Function Spaces
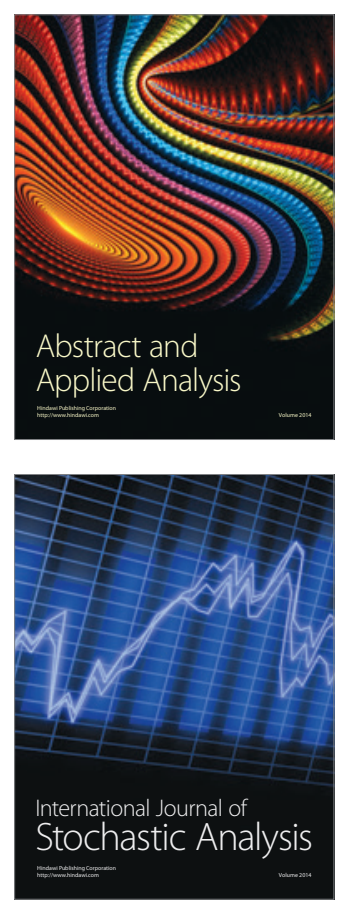

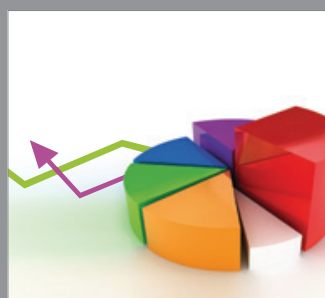

ournal of

Probability and Statistics

Promensencen
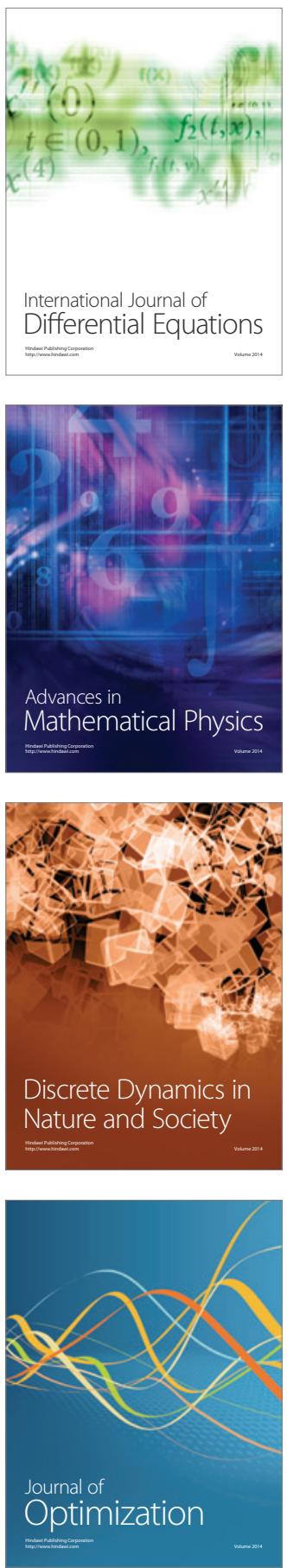